we are unaware of any information to suggest that time would affect the restenosis rate. As an alternate marker for time from onset of symptoms to revascularisation, we did find that treatment within 7 days of randomisation did not change the results, and restenosis rates were no different from those of patients treated more than 7 days after symptom onset.

We agree that early restenosis after carotid endarterectomy or stenting is probably due to neointimal hyperplasia, whereas atherosclerosis is the more frequent cause later on. Our analysis did not seek to identify individual causes for the restenosis lesions. However, this would be a potentially interesting investigation in subsequent studies.

Although there are no strong data to suggest the optimum treatment method for restenosis after carotid endarterectomy or stenting, a general clinical consensus has been emerging. An endovascular-first approach seems reasonable, and was the sole method used by the trial operators, even though this method was not mandated. Conversely, whether all such lesions need to be treated and what threshold of restenosis would mandate treatment are not yet known. Our own results, showing a higher stroke rate in patients with restenosis than in those without, argue on the side of repeat revascularisation.

We too are intrigued by the possibilities of pharmacological management of restenosis, considering some of the proinflammatory mediators associated with neointimal hyperplasia. Dyslipidaemia did indeed predispose to restenosis. However, CREST will not be in a position to test the hypothesis related to pleiotropic effects of statins, since almost all patients received lipid lowering therapy, and there was no statistical difference in use between the two groups of patients.

We declare that we have no conflicts of interest.
Brajesh K Lal, Thomas G Brott brott.thomas@mayo.edu

Division of Vascular Surgery, Department of Surgery, University of Maryland, Baltimore, MD, USA (BKL); and Department of Neurology, Mayo Clinic, Jacksonville, FL 32224, USA (TGB)

1 Lal BK, Beach KW, Roubin GS, et al, for the CREST Investigators. Restenosis after carotid artery stenting and endarterectomy: a secondary analysis of CREST, a randomised controlled trial. Lancet Neurol 2012; 11: 755-63.

\section{Disorders of \\ consciousness: are we ready for a paradigm shift?}

In their Personal View article in the August issue of The Lancet Neurology, Ralf Jox and colleagues discuss ethical and social aspects of requests from family members and surrogate decision makers for novel interventions in the clinical care of patients with disorders of consciousness (DOC). ${ }^{1}$ Functional imaging and neurophysiological approaches have been used to show awareness in some patients with DOC, despite clinical unresponsiveness. Jox and colleagues state that clinicians "must increasingly respond to requests by patients' families and surrogate decision makers to use novel techniques for diagnosis, prognosis, and treatment." This statement could be interpreted in several ways, and I am concerned that clinicians might draw the conclusion that such interventions should be offered as part of routine clinical care. The responses of clinicians to requests for the use of novel techniques have far reaching consequences for patients, members of their immediate family, doctors, and the legal profession.

To effect a clinical "paradigm shift"-in which novel imaging and neurophysiological tests are available in routine clinical care-researchers would need to provide convincing evidence of the need for change, and to show that such change in clinical practice would not be harmful to patients. However, apart from references to results published in highly specialised journals-the product of studies by a small number of investigators in the field-these important data were not provided by Jox and colleagues. Consequently, it is uncertain whether or not a paradigm shift is needed or whether research in this exciting field must be continued.

As discussed by the authors, clinicians have difficulty differentiating the minimally conscious state (MCS) from the vegetative state or unresponsive wakefulness syndrome. ${ }^{2}$ Investigators are assessing data on the natural history of these disorders, but our knowledge is far from complete. ${ }^{3}$ In patients in a MCS, investigators have described wilful modulation of the functional MRI (fMRI) signal ${ }^{4}$ in survivors of traumatic brain injury, but wilful modulation was not seen in patients with anoxic coma. This raises questions about the topography of brain injury in patients with traumatic brain injury and anoxic coma, and the residual brain network necessary to generate neurophysiological activity that can be detected in these tests. Such simple data could be invaluable to clinicians in predicting the type of patient who would have such responses on fMRI. For example, damage to the white matter tracts and the thalami on diffusion tensor imaging has been proposed to help in differentiating between persistent vegetative state and MCS. ${ }^{5}$ Unfortunately, these data linking the location of injury to fMRI or electrophysiological test results were not the focus of this Personal View. This lack of information makes it difficult for clinicians to decide when to call in help, and whom to call in, to do such tests.

Few groups have undertaken studies using the novel imaging and neurophysiological approaches that Jox and colleagues describe, which demand a good understanding of the relevant research methodology and the use of mathematical platforms

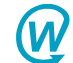

Published Online December 21, 2012 http://dx.doi.org/10.1016 S1474-4422(12)70289-2 


\section{Correspondence}

For FSL see http://fsl.fmrib.ox.ac uk/fsl/fslwiki/

For SPM see http://www.fil.ion. ucl.ac.uk/spm/

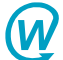

Published Online December 21, 2012 http://dx.doi.org/10.1016/ S1474-4422(12)70286-7 such as MATLAB (MathWorks, Natick, Massachusetts, USA), FMRIB Software Library (FSL), and Statistical Parametric Mapping (SPM) for the processing of functional images. I am not certain how many clinicians (neurologists and radiologists) are familiar with these analyses, which leads to the question of how generalisable these research methods are and to concerns about the potential harm to patients if such tests are not done properly.

Finally, what are the effects of these findings (modulation of brain activity) in patients with $\mathrm{DOC}$ on the patients and families involved in the research cited by Jox and colleagues? ${ }^{4}$ The authors state that requests for these interventions "provide an opportunity for clinicians to learn about patients' values and preferences", but I am not certain how this can be achieved in practice. One can never be truly certain of the values of patients with DOC, because this understanding would require communication beyond simple answers such as yes and no. When the authors say that clinicians should "maintain clinical acumen for changes in patient status with the patients' best interests in mind", do they mean that these patients should have regular MRIs every week for months or for years?

TGP has received honoraria as a speaker for Bayer and Genzyme.

\section{Thanh G Phan}

\section{thanh.phan@monash.edu}

Department of Neurology, Monash Medical Centre, 246 Clayton Road, Clayton Stroke and Aging Research Group, Department of Medicine, Monash University, Victoria 3168, Australia

1 Jox RJ, Bernat JL, Laureys S, Racine E. Disorders of consciousness: responding to requests for novel diagnostic and therapeutic interventions. Lancet Neurol 2012; 11: 732-38.

2 Kuehlmeyer K, Racine E, Palmour N, Hoster E, Domenico Borasio GD, Jox RJ. Diagnostic and ethical challenges in disorders of consciousness and locked-in syndrome: a survey of German neurologists. J Neurol 2012; 259: 2076-89.

3 Estraneo A, Moretta P, Loreto V, Lanzillo B, Santoro L, Trojano L. Late recovery after traumatic, anoxic, or hemorrhagic long-lasting vegetative state. Neurology 2010; 75: 239-45.
4 Monti MM, Vanhaudenhuyse A, Coleman MR, et al. Willful modulation of brain activity in disorders of consciousness. N EnglJ Med 2010; 362: 579-89.

5 Fernandez-Espejo D, Bekinschtein T, Monti MM et al. Diffusion weighted imaging distinguishes the vegetative state from the minimally conscious state. Neuroimage 2011; 54: 103-12.

\section{Authors' reply}

Thanh G Phan expresses scepticism about the clinical use of functional MRI (fMRI) in the care of patients with disorders of consciousness (DOC). In our Personal View article, we tried to discuss this issue in a careful and balanced way and we take this opportunity to clarify some of our statements to avoid possible misunderstandings.

First, the commentator seems to have misunderstood our use of the word "must" in the sentence he quotes at the beginning of his letter. By "must increasingly respond to requests", we meant that when families demand a test, we owe them an answer even if our answer is a denial of their request. We did not mean that neurologists must order any requested test, especially given the caveats about the accuracy, predictive value, methodological complexity, and availability of fMRI underscored in our article and discussed by Phan in his letter.

Second, we used the expression "potential paradigm shift" to explain that the traditional clinical assessment of DOC is increasingly recognised as insufficient to make an accurate differential diagnosis and a correct prognosis, both of which are necessary for adequate care. We argue that new imaging and neurophysiological tests as well as larger-scale datasets are needed to refine our diagnostic and prognostic methods. The high rate of clinical misdiagnosis is a finding that has been replicated in several studies that used different methods.
We emphasised that the syndromal difference between the unresponsive wakefulness syndrome (vegetative state) and the minimally conscious state is only one of several indicators for prognostication.

Third, we proposed that requests by patients' family members for further tests can be seen as an opportunity for communication about adequate care and treatment. Both ethically and legally, treatment depends on the patient's preferences. These preferences can be elicited by asking surrogates to help to interpret advance directives and to make substituted judgements, even if we can never be $100 \%$ sure in this respect. To apply the patient's preferences to the current clinical situation, it is essential to closely monitor the patient's clinical course and to be attentive to any positive or negative changes, which might occur even after long intervals. Because we need reliable technical tools to assess subtle changes in the condition of patients with DOC, we argue for further research in this direction accompanied by careful interdisciplinary reflection on the conditions for the use of these tools in clinical care.

$\mathrm{SL}$ is Research Director at the Belgian National Funds for Scientific Research (FRS). All other authors declare that they have no conflicts of interest.

\section{RalfJJox, James L Bernat,} Steven Laureys, Eric Racine ralf.jox@med.uni-muenchen.de

Institute of Ethics, History and Theory of Medicine, University of Munich, Munich, Germany (RJJ); Dartmouth Medical School, Hanover, NH, USA (JLB); Coma Science Group, Department of Neurology and Cyclotron Research Centre, University Hospital and University of Liège, Liège, Belgium (SL); and Neuroethics Research Unit, Institut de recherches cliniques de Montréal, Canada, Montréal, QC Canada (ER)

1 Jox RJ, Bernat JL, Laureys S, Racine E. Disorders of consciousness: responding to requests for novel diagnostic and therapeutic interventions. Lancet Neurol 2012; 11: 732-38. 